\title{
Introduction. Ce que les écrits font au travail
}

\author{
What writings do at work
}

Gwenaële Rot $^{\mathrm{a}, *}$, Anni Borzeix ${ }^{\mathrm{b}}$, Didier Demazière ${ }^{\mathrm{c}}$

a UMR 8533, institutions et dynamiques historiques de l'économie et de la société (IDHES), université de Paris Ouest Nanterre, 200, avenue de la République, 92001 Nanterre cedex, France

b UMR 7176, centre de recherche en gestion (CRG), école polytechnique, ENSTA Paris Tech, 828, boulevard des Maréchaux, 91762 Palaiseau, France

c UMR 7116 CNRS et Sciences Po, centre de sociologie des organisations (CSO), 19, rue Amélie, 75007

Paris, France

\begin{abstract}
Résumé
Les écrits, on le sait, circulent en abondance dans les milieux professionnels les plus divers. Aussi nombre de recherches portent-elles sur les formats, les usages et les rôles des écrits dans le travail. Mais la piste suivie ici ne vise pas à rendre compte de ce qui est fait des écrits dans le travail. L'hypothèse centrale repose sur une vision performative des écrits, qui leur prête un pouvoir d'agir, une agency. Elle peut se résumer ainsi : qu'est-ce que les écrits "font " au travail ? Étudier le travail par ses écrits permet d'en articuler deux dimensions trop souvent séparées : les activités situées d'une part, les fonctionnements organisationnels de l'autre. En effet, les écrits sont des traces matérielles et robustes sinon toujours pérennes, des activi-tés concrètes, et ils offrent une prise pour l'analyse rigoureuse du travail au plus près de ses conditions de réalisation. Mais les écrits voyagent et circulent. Ils tissent des liens entre travailleurs, groupes, ate-liers, services, firmes, sans lesquels la production ne pourrait pas aboutir. Ils étirent le travail. Les suivre, c'est donc déplacer l'enquête vers d'autres moments que le présent de la situation observée, vers d'autres espaces de travail aussi, à côté, aux alentours. Se révèlent alors les dimensions processuelle et interac-tive des organisations, leur propriété intégratrice. Ainsi, considérer ce que les écrits font au travail, ce n'est pas seulement se placer à un niveau intermédiaire entre celui de l'action située et celui de l'action organisée. C'est, pour le sociologue, la possibilité d'articuler « sur pièces » ces deux perspectives, en inscrivant l'analyse du travail concret dans des actions collectives plus larges, spatialement et temporellement, et en documentant l'analyse organisationnelle à partir des situations de travail observées. C'est propo-ser une contribution à une écologie de l'activité plus extensive, plus clairement arrimée à son enveloppe organisationnelle.
\end{abstract}

Mots clés : Écrits ; Travail ; Organisation ; Action située ; Action organisée

\footnotetext{
* Auteur correspondant.

Adresse e-mail : gwenaele.rot@orange.fr (G. Rot).
} 


\begin{abstract}
As is known, large numbers of writings circulate within every kind of professional environment. As a result, there is extensive research on the formats, uses and roles of writings in work. However, the approach taken here is not to describe what is done with writings in work. The central hypothesis rests on a performative vision of writings, one that gives them the power to act, agency. It can be summed up as follows: what do writings "do" to work? Studying work through its writings is a way to connect two dimensions that are too often kept separate: situated activities on the one hand, organizational functions on the other. Indeed, writings are material and robust - though not always lasting - traces of concrete activities, and they provide a handhold for the rigorous analysis of work as close as possible to its conditions of realization. However, writings travel and circulate. They form links between workers, groups, workshops, services, firms, without which production could not take place. They stretch work beyond its source. To track them is therefore to shift the field of enquiry to moments other than the present of the observed situation, and also to other nearby, surrounding workspaces. This brings out the procedural and interactive dimensions of organizations, their integrating capacity. Exploring what writings do to work is therefore not only to place oneself at an intermediate level between situated action and organized action. For the sociologist, it is a way of connecting these two perspectives "on paper", by embedding the analysis of the actual work in spatially and temporally broader collective actions, and by documenting organizational analysis on the basis of observed work situations. It is a way of contributing to a more extensive ecology of activity, more clearly anchored within its organizational envelope.
\end{abstract}

Keywords: Writings; Work; Organization; Situated Action; Organized Action

L'écriture, on le sait, n'est pas seulement une composante essentielle de multiples activités humaines : elle est aux sources mêmes de notre «raison graphique» (Goody, 1979). Plus spécifiquement, dans nombre de situations de travail et pour de nombreux métiers, il faut écrire pour accomplir ses tâches, il faut manier des écrits pour produire, il faut mobiliser des écrits pour penser, pour décider, pour agir. L'analyse des écrits dans le travail et de l'écriture au travail croise les études de la cognition au travail ${ }^{1}$ et des objets dans l'action. L'écrit apparaît dès lors comme une ressource cognitive mobilisée pour et dans le cours des activités. Il est aussi bien plus que cela.

Les écrits circulent en abondance en milieu professionnel et sous les formes les plus variées : tableaux et journaux de bord, courriers électroniques, notes de service ou notes griffonnées, documents administratifs, règlements, fiches de contrôle, agendas, relevés d'anomalies, listes de consignes, rapports d'activité, plannings, diaporamas pour présentations orales, checklists, postit, feuilles de brouillon, petits carnets personnels... Ils acquièrent ainsi des statuts différenciés : écrits officiels, écrits publics, écrits juridiques, écrits secrets, écrits «pour soi », écrits collaboratifs, écrits individuels, écrits «à plusieurs mains », écrits fugaces, écrits pérennes, écrits mobiles.

Les écrits du travail ont une identité ambiguë et confuse : ils «sont» plusieurs choses à la fois et remplissent, on le sait, des fonctions multiples - informer, contraindre, communiquer, transmettre, mémoriser, certifier, authentifier, prouver, sécuriser, surveiller, gouverner, notifier, conserver, conseiller, explorer, concevoir. Ils sont aussi bien des outils de la prescription, de la standardisation, de la formalisation, que des produits bricolés par ceux qui les utilisent pour

\footnotetext{
${ }^{1}$ Deux numéros de la revue ont rendu compte de ces travaux (voir en bibliographie: Sociologie du travail, 1994 et Sociologie du travail, 2008).
} 
travailler — dichotomie que l'on trouve souvent sous la plume des sociologues — ou encore des repères cognitifs pour l'activité, comme l'ont montré les recherches plus récentes, souvent rangées sous l'étendard des workplace studies (Luff et al., 2000).

Des travaux très diversifiés au plan des disciplines, des cadres théoriques, des méthodes, des terrains, des questions traitées ont examiné la place prise par les écrits - par des écrits chaque fois particuliers - dans le cours des activités de travail, ainsi que leur rôle dans l'action collective, son déploiement, son organisation. Plusieurs publications collectives récentes, livres ou revues, offrent des portes d'entrée propices à l'exploration de cet univers ${ }^{2}$ et indiquent un regain d'intérêt pour cette thématique. Elles portent notamment sur les «valeurs et enjeux des écrits de travail» (Semen, 2009), sur les «normes et écritures de l'organisation» (Études de communication, 2010), sur «les paradoxes de l'écriture » dans les institutions d'encadrement (Coton et Proteau, 2012), sur l'écriture des «petites mains de la société de l'information» (Revue d'anthropologie des connaissances, 2012), sur une anthropologie de l'écriture (Barton et Papen, 2010), sur les dispositifs de surveillance et de contrôle de l'alimentation (Bonnaud et Joly, 2012).

L'analyse des écrits a été conduite dans de nombreux métiers et secteurs professionnels $^{3}$ : bien sûr, ceux dont l'activité d'enregistrement est au cœur du travail au point d'en définir la fonction, tels les greffiers (Nouiri-Mangold, 2012), les sténodactylographes (Gardey, 2008), les écrivains publics (Nogard, 1997) et, dans ce numéro, les scriptes (Rot, 2014). Les professionnels de la santé, du milieu judiciaire ou du travail social (Lae, 2008), secteurs très «consommateurs» d'écrits, sont aussi bien étudiés: huissiers (Fraenkel et al., 2010), policiers (Levy, 1985; Proteau, 2012), inspecteurs du travail (Dodier, 1988), travailleurs sociaux (Delcambre, 1992; Serre, 2008), médecins et infirmières (Grosjean et Lacoste, 1998, 1999; Mathieu-Fritz et Guillon, 2008; Berg, 1996). Nombre de métiers de la «grande» administration ou de «la petite» - publique ou privée - ont été explorés à travers le prisme des écrits de travail: fonctionnaires des administrations centrales ou déconcentrées (Laurens, 2008; Torny, 2011; Bonnaud et Martinais, 2013), acteurs du gouvernement urbain (Hull, 2012), conseillers d'État (Latour, 2002), magistrats (Mouhanna, 2012), agents de gestion d'offices HLM (Pène, 1997), employés de banque (Denis, 2011). En lien avec le développement des exigences de traçabilité des process et des produits (Cochoy et al., 1998; Grandjou, 2003; Grandjou et Valceshini, 2005 ; Moatty et Rouard, 2010), les pratiques d'écriture des travailleurs de l'industrie et ceux de l'agriculture ont aussi fait l'objet de riches travaux: ouvriers de la sidérurgie (Charrasse, 1992), de la pétrochimie (Montredon, 1986), de l'automobile (Hatzfeld, 2002), des abattoirs (Muller, 2008), de I'agriculture (Joly, 2004 ; Joly et Weller, 2009; Mbodj-Pouye, 2008), ou encore auditeurs (Weller, 2012). On peut y ajouter encore bien d'autres mondes professionnels, comme ceux des travailleurs de la mer (Delcambre,

\footnotetext{
2 À partir de la sémio-linguistique, de la communication organisationnelle, de la sociologie, de l'anthropologie.

${ }^{3}$ De si nombreux univers professionnels que nous ne pouvons qu'en citer quelques exemples ici. Pour une enquête quantitative voir Moatty et Rouard (2010).
} 
2002 et, dans ce numéro, Flécher, 2014), des opérateurs de centres d'appels (Licoppe, 2002), des laborantins et chercheurs (Pontille, 2004; Rot, 2005; Brives, 2009), des enseignants (Lahanier-Reuter, 2010), des assistants parlementaires (Treille, 2007)... sans pouvoir clore I'inventaire.

Alors que les travaux existants informent sur la place (croissante ou non), sur les formes et les formats, sur les usages (ce que les salariés font des écrits ou ce que les prescripteurs font aux salariés avec leurs écrits) et sur le rôle des écrits dans le travail, nous voudrions ici emprunter une autre voie en nous demandant ce que les écrits «font» au travail. Ce questionnement s'appuie sur les acquis des recherches développées depuis les années 1990 par le réseau pluridisciplinaire Langage et travail (Borzeix et Fraenkel, 2001 ; Pène et al., 2001), mais il les prolonge en insistant sur les enseignements proprement sociologiques qu'offre aujourd'hui cette perspective pour l'analyse conjointe du travail et de l'organisation. Partant du constat désormais évident que le travail se fait non seulement avec des gestes mais aussi avec des mots, ces travaux ont montré tout l'intérêt qu'il y a à étudier la «part langagière du travail », une dimension par trop négligée dans les recherches en sciences sociales en général et en sociologie du travail en particulier. Cette prise en compte s'est concrétisée dans des travaux pionniers sur les pratiques d'écriture « quotidiennes » ${ }^{4}$ ou ordinaires au travail (Fraenkel, 1993a, 1993b, 2001 ; Boutet, 1993 ; Delcambre, 1997 ; Pène, 1997). Faut-il rappeler que si le travail se fait avec des mots, ces mots sont souvent des mots écrits, dont les supports se sont amplement développés avec l'extension des technologies informatiques?

Il ne s'agit pas ici de prolonger les analyses du travail d'écriture lui-même, ni de repérer ce que font les travailleurs à ou avec ces écrits — les négliger, les oublier, les tordre, les appliquer, ou les ignorer par défiance, résistance ou désintérêt, ou encore les inventer et les manipuler -, même si ces dimensions sont évidemment présentes dans l'ensemble des contributions de ce numéro. Il s'agit tout d'abord de mettre à profit cet avantage méthodologique décisif qu'a l'écrit sur l'oral (du moins pour le chercheur désireux d'appuyer ses analyses sur du «consolidé ») : celui de fournir des traces matérielles robustes, non fabriquées pour lui ou à sa demande, et qui résistent au temps. Faire de ces archives une source de connaissance pour la sociologie du travail est l'un des apports majeurs de ces travaux.

Il s'agit ensuite de constituer les écrits en analyseurs pour raisonner sur les connexions, scruter les flux, débusquer les dynamiques des activités professionnelles, ceci pour enrichir la description de ces formes contemporaines de travail qui accroissent les exigences de coordination. On sait, grâce à Pierre Naville (1963) notamment, que ces exigences sont un impératif productif ancien. Mais les occasions et contraintes de coordination, de concertation, de transmission, de négociation, de collaboration, entre les firmes, les services, les équipes et les salariés, se démultiplient avec le développement des organisations en réseaux, de l'éclatement des firmes, des sous-traitances en cascades, des rapports clients-fournisseurs entre services, des équipes composées de salariés rattachés à plusieurs employeurs, du travail à distance ou télétravail, des espaces collaboratifs, de la désynchronisation des activités, de la production en continu et de la rotation des équipes, de la multi-activité, etc. Ces phénomènes sont disparates et se diffusent de manière très inégale selon les secteurs d'activité. Ils conduisent pourtant à se demander comment la dispersion est gérée, la continuité produite, l'activité d'organisation déployée, et à s'interroger sur les alentours

\footnotetext{
${ }^{4}$ Nous empruntons l'expression au titre de l'ouvrage dirigé par Daniel Fabre (1997).
} 
du travail, sur ce qui se passe avant, après, ailleurs, autour et entre les postes, là où l'organisation en actes se fabrique "par en dessous », au cœur de ce processus de production-là.

Il s'agit enfin de mobiliser les écrits, toutes sortes d'écrits, dans la variété de leurs origines, de leurs supports, de leurs formes et de leurs usages, pour analyser le travail autrement. Étudier le travail à partir de ses écrits permettrait — tel est à notre sens l'un des enjeux théoriques de ce numéro - de reconnecter et réarticuler deux dimensions trop souvent séparées dans les analyses : les activités situées d'une part, les fonctionnements organisationnels de l'autre ${ }^{5}$. Nous y voyons l'opportunité d'une posture de l'entre deux, ni en surplomb ni trop au ras du sol, à même d'établir des jonctions entre ces deux perspectives ; une posture tentant ainsi de restituer à l'activité située les dimensions spatio-temporelles sans lesquelles elle manque de relief. Entrer par les écrits offre au chercheur la possibilité d'articuler «sur pièces » ces perspectives en inscrivant l'analyse sociologique du travail dans des actions collectives plus larges, afin de documenter l'analyse organisationnelle à partir des situations de travail observées. Le travail se présente alors, et cette formule n'est pas un jeu de mots, sous les traits d'un «raccord», un raccord dont la force et la forme sont spécifiques à chacun des cas étudiés. Les connexions placées au cœur même de l'observation invitent à se demander, preuves à l'appui en quelque sorte, entre quoi, comment, pour qui, pour quoi la connexion a lieu, et ceci grâce à un double mouvement.

D'un côté, l'attention aux écrits élargit l'espace-temps considéré. Sur la base de documents empiriques - les écrits sont des instruments de traçabilité pour tous, salariés, directions et chercheurs -, elle déplace certaines des limites spatio-temporelles qui ont marqué les travaux en sociologie du travail. Tout en poursuivant la direction ouverte par une sociologie du poste, de l'atelier puis de l'activité, les articles réunis ici donnent à voir un au-delà de la situation. Les écrits — du moins beaucoup d'entre eux — voyagent et circulent, ce qui invite à déplacer l'enquête, à en considérer les «alentours », ce qui se passe à côté, dans d'autres espaces de travail, mais aussi entre ces espaces. De même, les écrits perdurent et résistent au temps, ce qui amène à élargir l'examen à d'autres moments que le présent de la situation observée, à considérer ces autres «alentours » que constituent l'avant et l'après, les traces du travail et leur transmission au cours du temps.

D'un autre côté, les écrits sont considérés ici comme un «traceur » de l'organisation « réelle » du travail, qui voudrait dépasser la distinction bien connue entre travail prescrit et travail réel. Les écrits tissent, entretiennent et consolident les liens entre travailleurs, groupes, ateliers, services, firmes, sans lesquels la production ne pourrait aboutir. Aussi, les chemins pris par les écrits mettentils au jour la dimension processuelle et interactive des organisations, leur propriété intégratrice. Les écrits fournissent une prise empirique robuste, tangible, pour décrire finement des mécanismes —à l'échelle organisationnelle — qui demeurent souvent encore trop abstraits, comme la coordination, la coopération, la collaboration, la coproduction ou encore la distribution ${ }^{6}$.

Les contributions à ce numéro sont le fait de sociologues — et non de sociolinguistes — qui pour la majorité n'ont pas fait de l'écrit leur objet principal, mais ont néanmoins éprouvé le besoin, chemin faisant, de prendre l'écrit au sérieux afin de rendre compte plus rigoureusement des activités de travail étudiées. Elles livrent des données empiriques, inscrites chaque fois dans l'analyse d'un domaine d'activité et d'un métier précis : les scriptes sur un plateau de tournage (Gwenaële

\footnotetext{
${ }^{5}$ Cette séparation se traduit dans la distinction institutionnelle cinquantenaire entre sociologie du travail et sociologie des organisations, que ce numéro espère déconstruire au moins un peu.

${ }^{6}$ Concept partagé par la plupart des textes du numéro spécial de Sociologie du travail: «Travail et cognition II 》 (voir Borzeix et Cochoy, 2008).
} 
Rot), les marins dans un navire de commerce fonctionnant au tramping (Claire Flécher), les personnels de surveillance pénitentiaire dans un centre de rétention pour mineurs (Nicolas Sallée et Gilles Chantraine), les agents de maintenance de la signalétique dans le métro parisien (Jérôme Denis et David Pontille) et les spéculateurs en produits laitiers (Samuel Pinaud). Mais elles font plus que cela : ces descriptions obligent à déborder, chaque fois aussi, les limites du poste ou de l'activité examinée : suivre la piste des écrits représente une méthode heuristique pour renouveler nos prises analytiques sur des organisations déconcertantes, mouvantes, aux frontières labiles. Organisé autour d'interrogations différentes, enraciné dans les terrains explorés, chaque article peut être lu comme une contribution à cette problématique.

Prenons la réalisation d'un film (article de Gwenaële Rot), une activité qui comporte sa part de dispersion: le tournage est effectué en de multiples lieux; il est inscrit dans une temporalité discontinue, différente du déroulé du récit; il est marqué par de nombreux imprévus et par des controverses liées au tâtonnement créatif. L'organisation constituée pour la fabrication d'un film, «le projet», est, elle aussi, à sa manière, très fragile, en raison de la multiplicité des aléas à résoudre, des risques d'erreurs, des fortes contraintes temporelles. De plus, le tournage d'un film mobilise une multiplicité de professionnels (acteurs, maquilleurs, habilleurs, coiffeurs, cadreurs, etc.), dont les contributions doivent être agencées et coordonnées afin de garantir la cohérence du produit final, le film. C'est en particulier à la scripte - un métier fortement féminisé — qu'incombe la tâche de veiller à la «continuité du film», qui commande les conditions du montage et la vraisemblance de ce qui sera projeté sur l'écran.

Tout au long du tournage, la scripte s'appuie sur «son» scénario, soit un cahier dont elle ne se sépare jamais, qu'elle annote et enrichit en continu par des schémas, des dessins, des écritures abrégées. C'est d'abord une sorte d'aide-mémoire, auquel elle se réfère pour assurer le raccordement des séquences tournées. À ce scénario s'ajoute la feuille de continuité que la scripte a préparée et qui déroule les scènes du film dans l'ordre du tournage, suivant le plan de travail élaboré par la réalisation. Ces écrits ne renferment pas toutes les informations nécessaires pour garantir le bon déroulé du film, et le travail de la scripte s'appuie sur d'autres outils de mémorisation, tels le combo et l'appareil photo numérique. Suivre le travail de la scripte en s'intéressant à la façon dont, par l'écrit, elle supervise la continuité du film et débusque les problèmes de raccord en vue de contribuer à la fiabilisation de l'organisation productive du tournage, met au jour l'important travail d'ajustement entre différents corps de métiers que permet un «usage combinatoire » de l'écrit professionnel — un écrit au cœur de la définition de sa fonction. Mais le travail d'écriture a une performativité qui n'est pas dissociable du dense travail relationnel engagé sur le plateau, qui permet de faire circuler les informations pertinentes pour garantir la cohérence du film, de surveiller le travail des autres pour l'infléchir ou le rectifier en cas de nécessité. En produisant et mobilisant ses écrits, la scripte retisse une continuité sans cesse compromise par les conditions de réalisation. Elle agence différents horizons temporels, réagissant dans l'urgence du moment présent, mobilisant les propriétés de ce qui a été produit dans le passé, tout en se projetant dans le futur du film. L'analyse de son travail à partir de l'écrit, tel le scénario tout à la fois document professionnel pour soi et document d'organisation, permet alors d'éclairer le fonctionnement d'une organisation par projet, qui est aussi une «organisation sensible».

Dans l'analyse du fonctionnement d'un bateau de la marine marchande (article de Claire Flécher), le travail n'est pas appréhendé dans un cadre borné, spatialement comme temporellement. L'espace de travail est éclaté en de multiples lieux dans lesquels les membres d'équipage conduisent leurs activités : la salle des machines, la cabine de pilotage, le pont, etc. Mais il déborde bien au-delà de ces limites car le navire est constamment relié à la terre et à une multitude d'acteurs sans lesquels le commerce maritime ne pourrait s'effectuer: la compagnie propriétaire qui arme 
le bateau et lui fournit des contrats, le client affréteur qui indique la route maritime à suivre, les autorités des ports d'escale et des pays traversés qui donnent des autorisations d'accostage, etc. Le navire, à l'instar des centrales nucléaires ou des complexes pétrochimiques, est une organisation qui fonctionne en continu et sans rupture temporelle, rythmée par une multitude de scansions, régulières (la rotation des quarts) ou irrégulières (les cycles des manœuvres, des fluctuations météorologiques, des escales, des renouvellements d'équipages). L'attention portée aux écrits offre alors des prises pour analyser une organisation désarticulée, fluctuante.

Les écrits identifiés par Claire Flécher sont multiples et hétérogènes, dans leur forme comme dans leur circulation : cahiers et journaux de bord personnels, livres de bord (passerelle, machine et radio), code ISM (International Safety Management), checklist de procédures, rapports d'accident, documents de presque accident, etc. Tous contribuent à couturer et raccorder une organisation, et ce avant tout afin de la sécuriser. En effet, le navire concentre de nombreux risques (technologiques, environnementaux, économiques), ce qui renforce la nécessité de la production et de la circulation des écrits : des écrits codifiés permettent la communication entre navigants qui travaillent en décalé ; des flux continus d'échanges d'informations entre toutes les parties répondent aux exigences du «tout savoir en temps réel»; des enregistrements formalisés fournissent des traces de la conformité des actes à la réglementation, et constituent des pièces opposables pour l'imputation de responsabilités juridiques en cas d'incident ; des écrits plus personnels et informels permettent aux navigants d'avoir leur propres repères pour intervenir dans un contexte instable et fragile; des documents officieux, qui renforcent les échanges oraux, visent à attirer l'attention de la relève sur des particularités du navire ou du contexte de navigation, etc. L'attention portée aux écrits permet non seulement de décrire ces dispersions temporelles et spatiales, mais aussi de comprendre comment elles sont réduites à travers de multiples reconnections. Les écrits agencent l'organisation, car ils facilitent la coordination d'acteurs éparpillés, sur et hors du navire, et lui assurent une persistance à travers les traces qu'ils représentent. Ainsi, ils fonctionnent comme un dispositif de couture qui contribue à la confection de l'organisation. Ici, les écrits mobilisés par les navigants font apparaître la centralité de la question de la pérennité du navire, en tant que bâtiment naval soumis à de multiples aléas : une organisation productive «éclatée et désynchronisée ».

Le travail des surveillants des établissements pénitentiaires pour mineurs est également indissociable d'une activité d'écriture, une tendance accentuée par l'introduction d'un dispositif informatisé - le cahier électronique de liaison - fait pour consigner des observations quotidiennes (article de Nicolas Sallée et Gilles Chantraine). Analyser ce qui est consigné ou pas, et de quelle manière, comprendre ce qui vaut d'être tracé et enregistré, ou encore saisir les usages dont ces écrits font l'objet, constituent alors des objectifs de recherche susceptibles de renseigner le travail des gardiens : s'agit-il de surveiller, de protéger, d'alerter? Quels sont les indices et les observations qui déclenchent le passage à l'écrit? Quels sont les schèmes de perception et les catégorisations qui organisent la prise de notes? Support d'une évaluation rapprochée du comportement des détenus, l'écrit contribue aussi, dès lors qu'il est inscrit dans un cahier électronique connecté à un réseau informatique, à reconfigurer l'organisation du travail de surveillance des mineurs. Circulant entre surveillants, éducateurs de la protection judiciaire de la jeunesse, personnels de soins et enseignants intervenant en prison, ces écrits organisent la «prise en charge globale » des détenus : ce sont des outils de coordination.

Avec le cahier électronique, les traces des observations consignées deviennent accessibles à une pluralité de professionnels et elles sont insérées dans une temporalité élargie — au moins celle de la carrière d'incarcération du détenu. Des recherches portant sur le travail dans les institutions judiciaires ou sociales ont pu insister sur le développement de systèmes généralisés de surveillance, et y repérer le symptôme du caractère panoptique de certaines organisations. Mais 
on peut aussi voir dans la circulation électronique des écrits de travail le signe du déploiement de formes d'organisation feuilletées et éclatées, et la possibilité pour le chercheur de les appréhender. Car les écrits et leurs adressages multiples connectent des acteurs engagés dans des cours d'action distincts, dessinant ainsi les organisations effectives de travail. L'usage du cahier électronique ne supprime pas les transmissions verbales. Mais la forme écrite a son efficacité propre, puisqu'elle fixe les observations et limite les possibilités de redéfinir les cas, même si la mobilisation des informations consignées lors de réunions peut aussi bien contribuer à figer et durcir les catégorisations qu'à susciter de nouvelles interprétations ou encore à les mettre en débat. La circulation des écrits, particulièrement rapide et fluide dans le cas du cahier électronique, leste l'activité d'écriture. Le soin que mettent les surveillants de prison à noter des données, non seulement sur les détenus mais aussi sur la manière dont ils ont pris en charge la situation critique, est révélateur de cet enjeu. Si l'écrit est susceptible de discréditer les travailleurs lorsqu'il conduit à signaler un manquement dans l'exercice de l'activité, il peut aussi être utilisé pour mettre en valeur leurs compétences, ou comme dispositif de protection juridique (en cas de survenue d'incidents ou de risque de suicide d'un détenu). Les modes d'appropriation de l'écrit sont variés, et ce qu'ils font au travail est médiatisé par des usages qui peuvent aller de la reconnaissance d'une utilité jusqu'au discrédit, et qui sont liés à l'appréciation de ce qu'ils apportent pour l'accomplissement du travail.

Des enjeux similaires sont évoqués à propos des agents de la RATP chargés de l'entretien de la signalétique et des usages qu'ils font du «relevé», ce document qu'ils renseignent afin de déclencher le processus de réparation des anomalies (article de Jérôme Denis et David Pontille). En effet, dès lors qu'il est injecté dans une chaîne d'actions conduisant au remplacement des panneaux défectueux ou manquants, ce relevé peut aussi être utilisé comme source de contrôle des interventions des agents. Sous l'apparence anodine d'un moment parmi d'autres dans une succession d'activités elle-même soutenue par une chaîne d'écritures, le relevé fait plus que décrire avec précision (croquis, prise de cotes des panneaux, etc.) un désordre situé dans son environnement matériel. C'est un instrument de coordination indispensable pour assurer la séquentialité des interventions de maintenance : il résulte d'une opération préalable de détection et de signalement d'un désordre, et il inaugure une série d'autres opérations, comme la commande d'un nouveau panneau, sa fabrication et son installation. Cette coordination séquentielle est aussi un agencement d'interventions de différents services dans une entreprise multi-sites telle que la RATP.

La pratique du relevé est en quelque sorte une enquête que l'organisation dispersée fait sur elle-même pour que le processus d'ordonnancement puisse dérouler comme prévu les étapes de la procédure. L'espace-temps du relevé relie ce que la division du travail sépare, et peut se lire comme la trame écrite de l'organisation «réelle»: il trace, si on sait les traquer, les raccords qui articulent ensemble les morceaux épars de ce vaste système organisé. Et les statuts changeants endossés par le relevé au cours du circuit de la réparation (fiche d'intervention carbonée complétée à partir des relevés, enregistrement informatique de l'opération, codification par la saisie dans un progiciel spécialisé, passation d'une commande auprès d'un prestataire, etc.) font de cet écrit du travail le témoin actif de la pluralité des registres de la coordination dont la maintenance a besoin pour assurer sa propre continuité.

Le travail des spéculateurs en produits laitiers offre un dernier terrain pour le repérage des effets structurants des écrits sur le travail, sur les activités réalisées au poste, c'est-à-dire ici devant des écrans d'ordinateur et au téléphone, comme sur l'organisation marchande des échanges commerciaux (article de Samuel Pinaud). Dans cet exemple comme dans celui de la marine marchande, la mondialisation transforme les conditions d'exercice du métier. Mettant en œuvre le conseil de Saskia Sassen qui pousse les sociologues à étudier de manière précise les «lieux 
centralisés où le travail de globalisation est accompli» (Sassen, 2009), Samuel Pinaud s'efforce de comprendre comment ces traders répondent à la question « où est le marché ?» : comment fontils pour estimer le prix auquel ils pourront vendre la marchandise de leurs clients ? Ce marché a deux caractéristiques saillantes : il est dépourvu d'une organisation centralisée qui fournirait des repères en matière de prix (comme les cotations officielles sur les marchés boursiers) et il exige une grande réactivité en raison notamment du caractère périssable des produits échangés. Il convient donc de réaliser des appariements dans des temporalités courtes, et ceci alors que les marges dégagées sont très faibles. L'évaluation anticipée des prix de revente et l'estimation des opportunités de cession de la marchandise acquise sont donc deux composantes importantes du travail de spéculation.

Devant s'orienter sur un marché qui manque de représentations marchandes «clés en mains », les spéculateurs produisent des écrits qui les aident à enregistrer et mémoriser les décisions prises et qui constituent en cela des appuis cognitifs à leur travail. Ces écrits sont aussi des matérialisations du marché ; ils sont les vecteurs du travail marchand. Les stratégies spéculatives s'appuient sur deux sortes d'écrits. Les uns, au contenu elliptique (notes personnelles consignées sur un cahier), sont les supports de leur rapport quotidien au marché en train de se faire - savoir interpréter les chiffres qui comptent. Ils restent à la discrétion de leurs propriétaires qui y inscrivent la valeur des produits, l'état des offres et demandes, les opportunités d'affaire, autant d'informations destinées à anticiper les fluctuations de prix. Ces écrits répondent aux exigences de l'activité (dégager des marges) et à ses contraintes (le temps compressé). Les autres écrits (feuilles volantes et tableaux informatiques) sont d'un usage plus collectif et renseignent sur la dimension distribuée de la spéculation. Ils matérialisent les engagements oraux, passés au téléphone, et sont les déclencheurs de l'intervention des logisticiens, acteurs à part entière de la spéculation. Celle-ci est en effet inséparable de l'activité de transport et de livraison, car elle porte sur des marchandises matérielles et non sur des titres dématérialisés qui circuleraient par le seul truchement de jeux d'écriture. Ici l'écrit et sa circulation connectent des composantes inséparables, mais pourtant dissociées par la division du travail, de l'activité spéculative. Et c'est grâce aux traces — lilliputiennes — de cette inscription qu'un accès aux épreuves jalonnant le processus - très global — de financiarisation des marchés agro-alimentaires est ménagé. Les écrits donnent accès au travail spéculatif, depuis la production de représentations opératoires du marché des produits laitiers jusqu'à l'organisation de la prise en charge matérielle de ces marchandises.

À partir d'études de cas, ces cinq articles montrent que les écrits «étirent» le travail et captent ses ramifications : le travail qu'ils donnent à voir déborde du cadre dans lequel l'analyse minutieuse des pratiques tend à le circonscrire, et relie entre elles des activités distantes aux plans spatial et temporel. Ils livrent du travail une version «enchaînée », mais dans un tout autre sens qu'habituellement : celui d'une activité prise dans «sa» chaîne de production, dont elle n'est qu'un maillon; une activité connectée, ancrée dans un système productif qui est organisé entre autres à travers la circulation, les usages et la transformation des écrits. Mais ces contributions ne sont pas focalisées de manière exclusive sur les écrits : elles les considèrent dans leurs relations et combinaisons avec d'autres artefacts, l'appareil photo ou le casque retour son de la scripte, la radio pour les marins, la caméra de surveillance des gardiens de prison, le progiciel spécialisé de commande pour la maintenance, les outils informatiques de calcul ou le téléphone du trader, etc.

Finalement, ce dossier voudrait proposer une contribution à une écologie de l'activité plus « extensive», plus clairement arrimée à son enveloppe organisationnelle, plus soucieuse de ses articulations avec un espace-temps qui l'englobe, de manière à voir dans cet ensemble qui lui sert de contexte autre chose qu'un simple lieu, un plateau de tournage, un navire, une station de métro, une salle de marché, etc. Ce que livre une observation empirique attentive aux connexions 
établies par les écrits du travail ne peut être qu'une «prise » incomplète sur cet ensemble organisé et en acte dont on suit ici, méticuleusement, le parcours de la consolidation, que l'on voie dans cet ensemble un produit (un film), un secteur (la marine marchande), une institution (le système pénitentiaire), une entreprise (la RATP) ou un marché (agro-alimentaire). Parler d'une perspective connexionniste mériterait développement et discussion, mais tel est bien l'horizon intellectuel esquissé. Par la mise en évidence de ce travail raccordé, émerge avec lui la part d'organisation dont il dépend, certes, mais aussi qui lui revient : une organisation capillaire et orchestrée, fragile et stabilisée, faite d'activités dispersées, interconnectées et néanmoins pilotées. C'est entre ces tensions que circulent les écrits.

\section{Déclaration d'intérêts}

Les auteurs déclarent ne pas avoir de conflits d'intérêts en relation avec cet article.

\section{Références}

Barton, D., Papen, U. (Eds.), 2010. The anthropology of writing. Continuum, London.

Berg, M., 1996. Practices of reading and writing: the constitutive role of patient record in medical work. Sociology of Health and Illness 18 (4), 499-524.

Bonnaud, L., Martinais, E., 2013. Écrire la loi. Un travail de bureau pour hauts fonctionnaires du ministère de l'écologie. Sociologie du travail 55 (4), 475-494.

Bonnaud, L., Joly, N. (Eds.), 2012. Alimentation sous contrôle. Tracer, auditer, conseiller. Éditions Quae et Educagri, Paris.

Borzeix, A., Cochoy, F., 2008. Travail et théories de l'activité : vers des workspace studies ? Sociologie du travail 50 (3), 273-286.

Borzeix, A., Fraenkel, B. (Eds.), 2001. Langage et travail. Communication, cognition, action. CNRS Éditions, Paris.

Boutet, J., 1993. Écrits au travail. In: Fraenkel, B. (Ed.), Illettrismes. Variations historiques et anthropologiques. BPI-Centre Georges Pompidou, Paris, pp. 253-266.

Brives, C., 2009. Le rôle des écrits éphémères dans la production des faits scientifiques. La domestication de la levure sauvage. Langage et société 127 (1), 71-81.

Charrasse, D., 1992. L'usine, l'écriture et la place. Savoirs techniques dans les transports sidérurgiques (1954-1989). Genèses 7, 63-93.

Cochoy, F., Garel, J.P., de Terssac, G., 1998. Comment l'écrit travaille l'organisation : le cas des normes ISO 9000. Revue française de sociologie 39 (4), 673-699.

Coton, C., Proteau, L. (Eds.), 2012. Les paradoxes de l'écriture. Sociologie des écrits professionnels dans les institutions d'encadrement. Presses Universitaires de Rennes, Rennes.

Delcambre, P., 1992. Écrire: conditions de travail et place professionnelle. Études de communication 13, 47-72.

Delcambre, P., 1997. Écriture et communications de travail. Presses Universitaires du Septentrion, Paris.

Delcambre, P., 2002. Un salarié a-t-il un «rapport à l'écriture»? Quelques réflexions sur une activité des officiers de la marine marchande : remplir un journal de passerelle. Pratiques 113-114, 68-82.

Denis, J., 2011. Le travail de l'écrit en coulisses de la relation de service. Activités 8 (2), 32-52.

Dodier, N., 1988. Les actes de l'inspection du travail en matière de sécurité : la place du droit dans du droit dans la justification des relevés d'infraction. Sciences sociales et santé 6 (1), 7-28.

Études de communication, 2010. Normes et écriture de l'organisation 34.

Fabre, D. (Ed.), 1997. Par écrit. Ethnologie des écritures quotidiennes. Éditions de la Maison des sciences de 1'homme, Paris.

Flécher, C., 2014. Écrire l'incertitude. Le travail à bord des navires de commerce entre stabilisations, prises de risques et responsabilisations. Sociologie du travail 56 (1), http://dx.doi.org/10.1016/j.soctra.2013.12.019.

Fraenkel, B., 1993a. Le partage de l'énonciation dans les écrits de travail. Cahier Langage et travail 5, 61-78.

Fraenkel, B., 1993b. La traçabilité, une fonction caractéristique des écrits de travail. Cahier Langage et travail 6, 26-38.

Fraenkel, B., 2001. La résistible ascension de l'écrit au travail. In: Borzeix, A., Fraenkel, B. (Eds.), Langage et travail, communication, cognition, action. CNRS Éditions, Paris, pp. 113-142. 
Fraenkel, B., Pontille, D., Collard, D., Deharo, G., 2010. Le travail des huissiers : transformations d'un métier de l'écrit. Octarès, Toulouse.

Gardey, D., 2008. Écrire, calculer, classer. Comment une révolution de papier a transformé les sociétés contemporaines (1800-1940). La Découverte, Paris.

Goody, J., [1977] 1979. La raison graphique. La domestication de la pensée sauvage. Éditions de Minuit, Paris.

Grandjou, C., 2003. La traçabilité, un dispositif de confiance dans les activités techniques. L'exemple de la filière viande bovine. Cahiers internationaux de sociologie 115, 327-342.

Grandjou, C., Valceshini, E., 2005. L'extension de la traçabilité dans le secteur agro-alimentaire. Terrains et travaux 2 (9), 73-89.

Grosjean, M., Lacoste, M., 1998. L'oral et l'écrit dans les communications de travail ou les illusions du «tout écrit». Sociologie du travail 40 (4), 439-461.

Grosjean, M., Lacoste, M., 1999. Communication et intelligence collective. Le travail à l'hôpital. PUF, Paris.

Hatzfeld, N., 2002. Les gens d'usine. 50 ans d'histoire à Peugeot Sochaux. Éditions de l'atelier, Paris.

Hull, M.S., 2012. Government of Paper: The Materiality of Bureaucracy in Urban Pakistan. University of California Press, Berkeley.

Joly, N., 2004. Écrire l'événement : le travail agricole mis en mémoire. Sociologie du travail 46 (4), 511-527.

Joly, N., Weller, J.-M., 2009. En chair et en chiffres. La vache, l'agriculteur et le contrôleur. Terrain 53 (2), $140-153$.

Lae, J.-F., 2008. Les nuits de la main courante. Écritures au travail. Stock, Paris.

Lahanier-Reuter, D., 2010. Livrets de bord et travail des enseignants du second degré. Langage et société 134 (4), $55-78$.

Latour, B., 2002. La fabrique du droit. Une ethnographie du Conseil d'État. La Découverte, Paris.

Laurens, S., 2008. Les agents de l'État face à leur propre pouvoir. Éléments pour une micro-analyse des mots griffonnés en marge des décisions officielles. Genèses 72, 26-41.

Levy, R., 1985. Scripta manent: la rédaction des procès-verbaux de police. Sociologie du travail 27 (4), 408-423.

Licoppe, C., 2002. Le traitement des courriers électroniques dans les centres d'appels. Sociologie du travail 44 (3), $381-400$.

Luff, P., Hindmarsh, J., Heath, C., 2000. Workplace Studies: Recovering Work Practice and Informing System Design. Cambridge University Press, Cambridge.

Mathieu-Fritz, A., Guillon, A., 2008. Quand les patients hospitalisés (re)deviennent des personnes. La mise en place de journaux de bord dans un service de réanimation. Réseaux 151, 91-137.

Moatty, F., Rouard, F., 2010. L'écrit au travail et ses déterminants chez les salariés en France en 2005. Travail et Emploi 122, 39-52.

Mbodj-Pouye, A., 2008. Pages choisies. Ethnographie du cahier d'un agriculteur malien. Sociologie et sociétés XL (2), 96-108.

Montredon, R., 1986. Opérateur de process : 1'article indéfini ? Technologies, idéologies, pratiques 6 (3), 11-23.

Mouhanna, C., 2012. De la plume aristocratique à la plume gestionnaire. Le cas de la magistrature. In: Coton, C., Proteau, L. (Eds.), Les paradoxes de l'écriture. Sociologie des écrits professionnels dans les institutions d'encadrement. coll. «L lien social », Presses Universitaires de Rennes, pp. 85-104.

Muller, S., 2008. À l'abattoir. Travail et relations professionnelles face au risque sanitaire. Éditions de la Maison des sciences de l'homme et Éditions Quae, Paris.

Naville, P., 1963. Vers l'automatisme social ? Problèmes du travail et de l'automation. Gallimard, Paris.

Nogard, A.-V., 1997. Chez l'écrivain public: l'écriture par délégation. In: Fabre, D. (Ed.), Par écrit. Ethnologie des écritures quotidiennes. Éditions de la Maison des sciences de l’homme, Paris, pp. 125-144.

Nouiri-Mangold, S., 2012. Se distinguer dans un espace standardisé. Des greffières entre l'oral et l'écrit. In: Coton, C., Proteau, L. (Eds.), Les paradoxes de l'écriture. Paris Presses Universitaire de Rennes.

Pène, S., 1997. Lettre administrative et espace social. In: Fabre, D. (Ed.), Par écrit. Ethnologie des écritures quotidiennes. Éditions de la Maison des sciences de l'homme, Paris, pp. 201-217.

Pène, S., Borzeix, A., et Fraenkel, B. (Eds.), 2001. Le langage dans les organisations, une nouvelle donne. L'Harmattan, Paris.

Pontille, D., 2004. La signature scientifique : une sociologie pragmatique de l'attribution. CNRS Éditions, Paris.

Proteau, L., 2012. Scribes ou scribouillard. Les ambivalences de l'écriture dans la division du travail policier. In: Coton, C., Proteau, L. (Eds.), Les paradoxes de l'écriture. Sociologie des écrits professionnels dans les institutions d'encadrement. Presses Universitaires de Rennes, pp. 41-66.

Revue d'anthropologie des connaissances, 2012. Les petites mains de la société de l'information. Revue d'anthropologie des connaissances $6(1)$. 
Rot, G., 2005. Le Knowledge Management et l'économie du partage des connaissances. Propos sur un désinvestissement de forme. Économie et société, série travail 25 (4), 675-698.

Rot, G., 2014. Noter pour ajuster. Le travail de la scripte sur un plateau de tournage. Sociologie du travail 56 (1), http://dx.doi.org/10.1016/j.soctra.2013.12.017.

Sassen, S., 2009. La globalisation. Une sociologie. Gallimard, Paris.

Semen, 2009. Valeurs et enjeux des écrits de travail. Semen 28.

Serre, D., 2008. Une écriture sous surveillance : les assistantes sociales et la rédaction du signalement d'enfant en danger. Langage et société 126, 39-56.

Sociologie du travail, 1994. Travail et cognition. Sociologie du travail 36 (4).

Sociologie du travail, 2008. Travail et cognition II. Sociologie du travail 50 (3).

Torny, D., 2011. L'écriture administrative des circulaires: autorégulation professionnelle, produit d'expertise technique ou norme juridique? L'exemple des risques iatrogènes liés aux prions. In: Bessy, C., Delpeuch, T., Pélisse, J. (Eds.), Droit et régulations des activités économiques: perspectives sociologiques et institutionnalistes. LGDJ, Paris, pp. $255-268$.

Treille, É., 2007. Écrire par délégation. Pratiques d'écriture des assistants parlementaires de députés socialistes. Mots - les langages du politique 85, 97-106.

Weller, J.-M., 2012. L'époisses sous contrôle papier. In: Bonnaud, L., Joly, N. (Eds.), Alimentation sous contrôle. Tracer, auditer, conseiller. Éditions Quae et Educagri, Paris, pp. 43-59. 